CLAWAR 2020: $23^{\text {rd }}$ International Conference on

Climbing and Walking Robots and the Support

Technologies for Mobile Machines,

Moscow, Russian Federation, 24-26 August 2020.

https://doi.org/10.13180/clawar.2020.24-26.08.p01

\title{
RUSSIAN ROBOTICS: STATE OF TODAY, VIEW FOR THE FUTURE
}

\author{
IVAN L. ERMOLOV \\ Ishlinsky Institute for Problems in Mechanics RAS \\ Scientific Council on Robotics and Mechatronics of Russian Academy of Sciences \\ Prospekt Vernadskogo 101-1, Moscow, 119526, Russia \\ E-mail:ermolov@ipmnet.ru \\ http://ipmnet.ru/en/
}

\begin{abstract}
Russia started its first experience in robotics in 1930s. But its highlight was in 1970s with first moon rovers - Lunokhod. Now Robotics is recognized as one of key technologies in Russia. National robotics program covers essential part of modern research areas of robotics. However now it's a good point to target those research areas which will be crucial for robots of tomorrow.

This presentation will give an overview of the main research actors of Russian robotics and present Russian view for Robotics research for the future. Some questions of future research in area of mechanics and control of robots, as well as specifics of robots' applications will be discussed. These materials can be also used as guidelines for building international cooperation with Russian roboticists.
\end{abstract}

\section{The Beginning}

First Russian/Soviet design was undertaken in 1930's. There was a an attempt to create a series of teleoperated tanks. Each system contained a couple of commander tank with operator onboard and a tank executor. Tank executor could carry explosive, shoot from machine-gun, shoot flame-thrower. There have been produced up to 130 teleoperated tanks by the beginning of WWII. However communication link among commander tank and tank executor was a weak element of a system and further studies of these robots was delayed to later 1980s.

An enormous success was achieved by a Soviet moon-rover program "Lunokhod" in 1970's (fig. 1). That time there have been launched 2 almost similar rovers "Lunokhod-1" (Nov. 1970) and "Lunokhod-2" (Jan. 1973) both produced by VNII Transmash. Each of the rovers was controlled by crew of operators from Earth basing on images received from TVcameras. Rovers were equipped by sets of measuring sensors to study lunar soil and terrain, radiation level, panoramic photometers and special reflector to measure distance between Earth and Moon. Each crew contained 5 crew members. "Lunokhod" mission can be considered as a big success in that time as their paths length (10,5 and $42 \mathrm{~km}$ respectively) was achieved by another space rover mission only 40 years later during "Opportunity" mission.

Late 1970's have become a breakthrough for Russian/Soviet industrial robotics. There has been done an intensive attempt to bring robots in Soviet industry. Soviet factories have produced about 100,000 industrial robots of various types, which was almost $40 \%$ of all robots installed in industry around the world. However frankly speaking this breakthrough have not affected Soviet industry as strong as it was expected. Main reasons of the failure was a fragmented introduction of robots in factories, lack of coordination and strategic planning in robotization and, final but not least, unsatisfactory reliability level of robots [5]. This together with new economic changes in Russia in 1990's has lead to extinguishment of robots from Russian industry (except car manufacturing). 


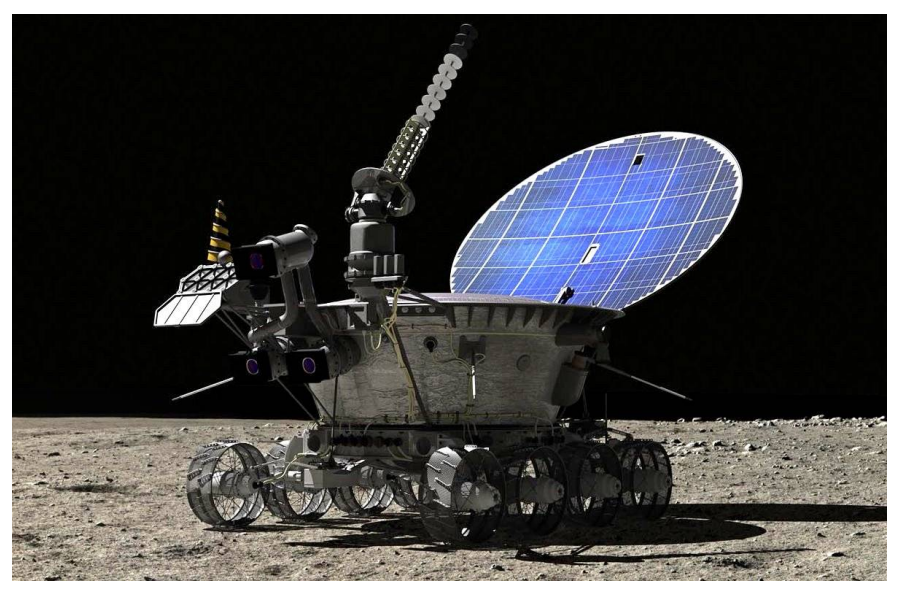

Fig. 1. "Lunokhod" moon-rover.

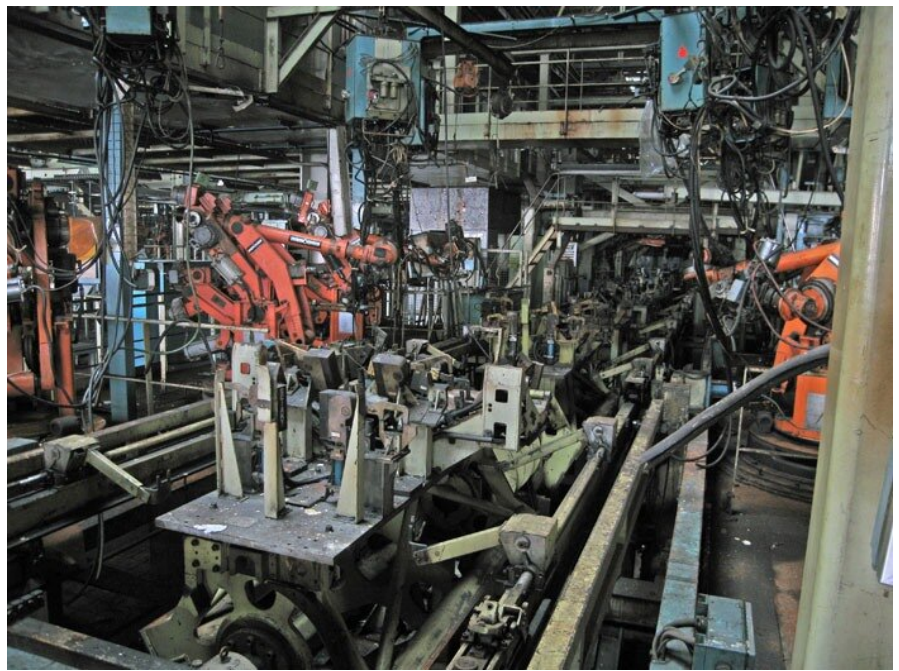

Fig. 2. Robots producing "Moskvich" cars.

Another stimulus for development of robots in Russia was a Chornobyl nuclear power plant in 1986. During catastrophy suppression there was a task to collect radioactive debris on the plant's roof and move it into building via breach of the roof. A special challenge was a very high radioactivity level which has led to malfunction of regular microelectronic components. For this task several robots were constructed by VNIITransmash, Bauman University and TcNII RTC. In total about several These robots were successfully used to clean radioactive debris and to explore territory unavailable to humans because of high radiation.

Transformations in Russian society and economy in 1990's have caused a slowdown in development of national robotic programme. Most of robots in industry were dismantled, national space programme has suffered severe cuts.

However "Nothing so bad, as not to be good for something" - due to experience of suppression of Chornobol nuclear power plant Russian Federation has always put a eye on robots for nuclear industry and high terrorist situation on Northern Caucasus has given strong stimulus for robots to by used by anti-terrorism forces. 


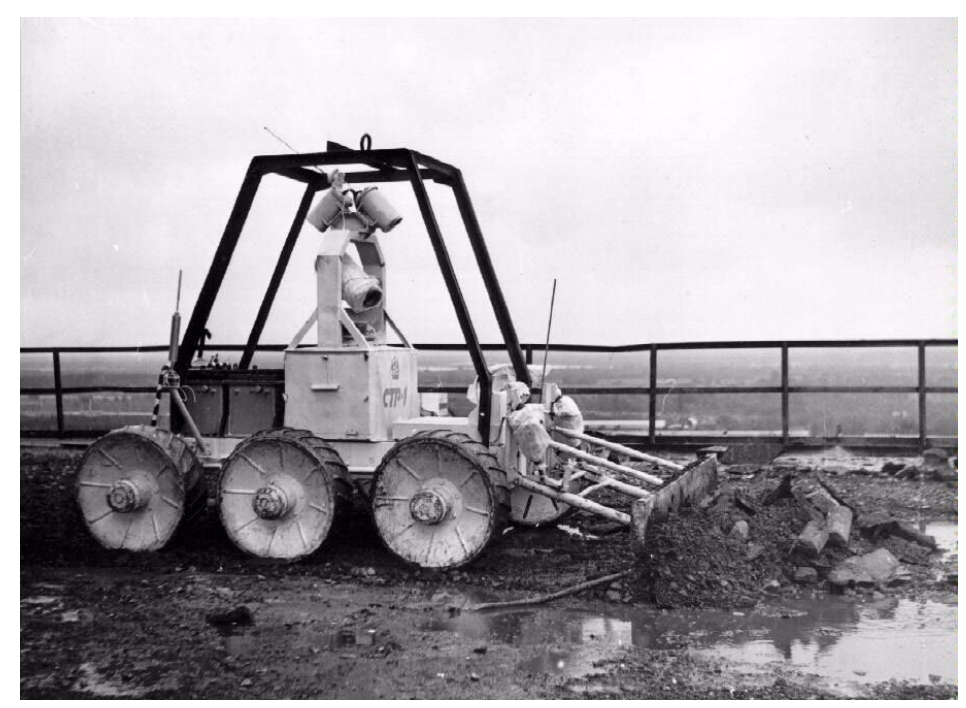

Fig. 3. STR-1 robot by VNII Transmash on roof of Chornobol nuclear power plant.

New political changes of 2000's have brought up an agenda of reviving Russian robotics programme.

\section{Later achievements}

Lately robotics have been recognized by Russian authorities a priority area of research. This creates an impact to strengthen robotics research in challenging areas. Here most interesting results are presented.

Fighting terrorism can not be performed without modern effective robots. Russian Federal Security Service for over then 15 years uses multifunctional robotic complexes "Vezdekhod" and "Varan" (fig. 4). These robots were designed and produced by a consortium of Bauman University and Kovrov Electro Mechanical Factory. Each of the models was equipped with rich set of sensors, hydrodynamic explosives disruptor and a set of supplementary equipment. In total more several hundreds copies of these robots were produced. Nowadays these robots are used all over Russia.

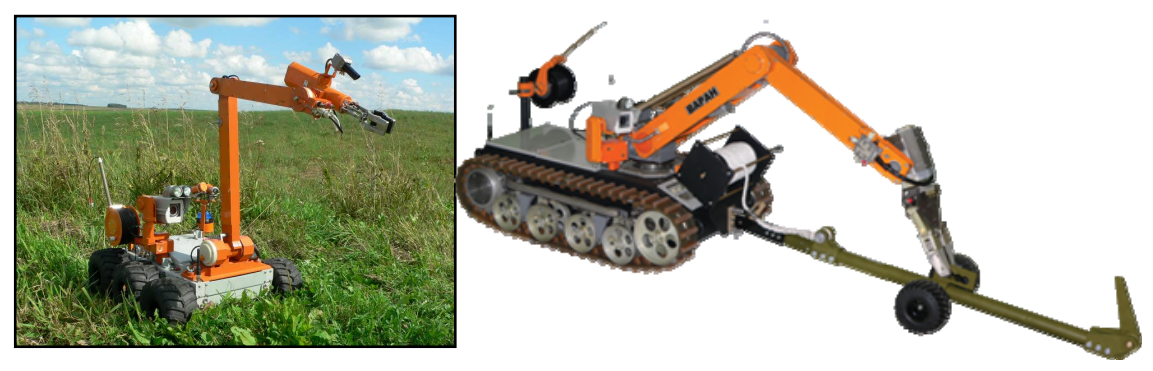

Fig. 4. "Vezdekhod" and "Varan" robots for explosives detection and disposal.

Nuclear industry is supplied by both robots for emerging situations (mostly by VNIIA) and by robots for everyday usage. Very impressive results were received by using robot for manipulation with fuel elements at nuclear power stations (Fig. 5). These robots are used to manipulate fuel elements during planned services of nuclear reactors. They were designed by a 
consortium coordinated by Southern Federal University. Experience of operating these robots has proved to considerably decrease service duration of nuclear reactor and as a result has increased effectiveness of energy production. Several dozens of these robots have been installed in nuclear power stations in Russia and abroad.

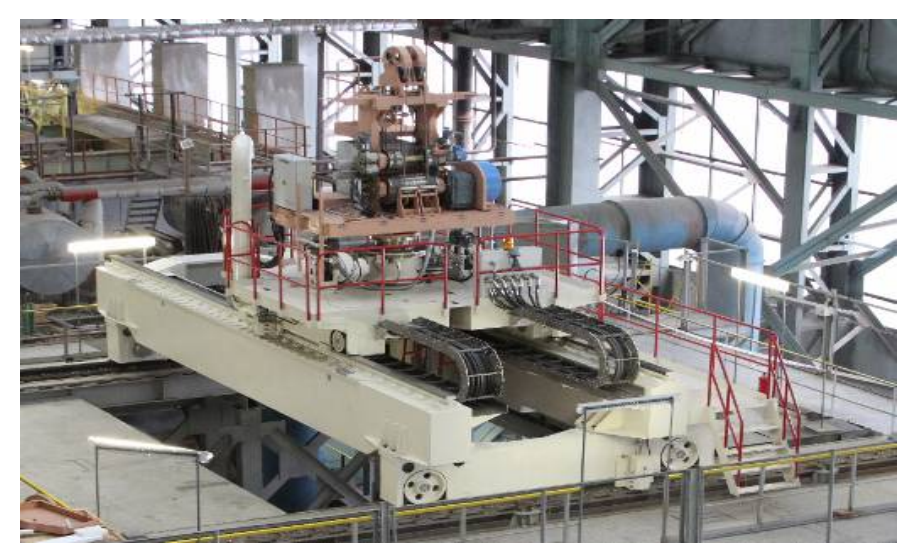

Fig. 5. Robot for manipulation with fuel elements.

Moscow based company "Taris" produces robots-crawlers for pipes inspection and repair (Fig. 6). Starting 1992 these robots are being used by municipal water services in Russia and abroad.

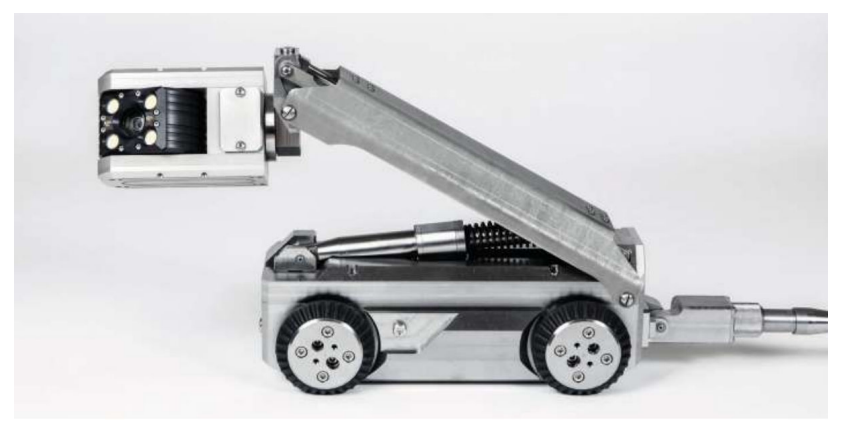

Fig. 6. Robot for pipes inspection by Taris.

As for industrial robotics one should mention industrial robots designed and produced by Autovaz and later by VMZ Inc (fig. 7).

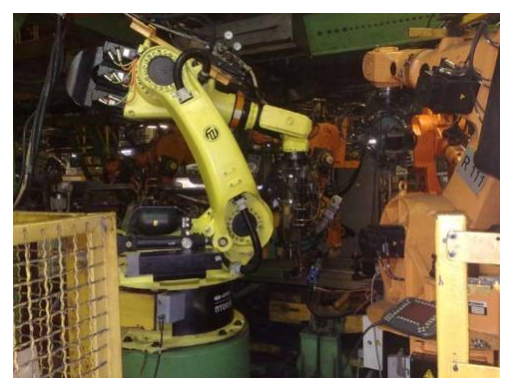

Fig. 7. Industrial robots by VMZ. 
In total almost thousand of robots of a popular in car manufacturing payload 50, 150 and $300 \mathrm{~kg}$ were produced. These robots were installed all over Russia mostly at car manufacturing companies.

\section{Main players}

In this section we present may players in Russian robotics world. We will mention their specialization in robotics.

Russian Academy of Sciences (RAS). Actually this is a unique form of organization of scientific research. RAS coordinates fundamental research of about 400 research institutes all over Russia. It is considered to be the main national consulting body of Russian Federation in scientific area. A prominent specifics of RAS is its multidisciplinarity. For better coordination research institutes group in 13 thematic sections and 3 regional sections. Because of this RAS is able to accumulate research efforts from various sciences. Main robotics activities in RAS are performed by Scientific Council for Robotics and Mechatronics of Russian Academy of Sciences. Council contains about 44 roboticists researchers and end-users. It implements various expertise in robotics, makes prognosing, strategic planning etc.

Ishlinsky Institute for Problems in Mechanics of Russian Academy of Sciences (IPMech RAS). The Institute is considered to have prime national expertise in area of climbing robots [12]. Overall there have been designed about 10 various climbing robots for maritime facilities, nuclear power stations, emercom etc. In 2020 a new test-field for climbing robots testing has been installed in Institute. Nowadays Institute also studies capsular robots, novel locomotion systems, exoskeletons, manipulative robots, data fusion [4] etc.

Trapeznikov Institute of Control Sciences of Russian Academy of Sciences. This institute has a high reputation in area of robotics control. Its main achievements are in: high-precision navigation of robots, UAV control, collaborative robots, cyber security in robotic systems. One should also mention a new test-field for UMVs, UAVs and indoor UGVs.

Keldysh Institute of Applied Mathematics Russian Academy of Sciences. Within this institute there are several laboratories who perform research in are of robotics. Among main scientific interests we should mention machine vision, exoskeletons, unmanned vehicles, sensor fusion.

St. Petersburg Institute for Informatics and Automation of Russian Academy of Sciences (SPIIRAS). As one may see from institute's title its main specialization is infromatics. Hereafter SPIIRAS has good expertise in multi agent control, cyber physical systems interaction and control of android-type robots [8].

Mechanical Engineering Research Institute of the Russian Academy of Sciences. This institute has a prominent expertise in area of manipulators built with a closed kinematic loop [7]. Another challenging area is using industrial robots for contact technological operations (e.g. metal cutting).

Institute of Marine Technology Problems of Far Eastern Branch of Russian Academy of Sciences is a key-player in marine robotics in Russia. Actually till recent time it was the only national manufacturer of heave-load UMVs in Russia. Nowadays it keeps being a leading fundamental research institute in area of underwater robotics, UMV control [11], UMV modeling.

Russian State Scientific Center for Robotics and Technical Cybernetics. This institute has from its beginning space robotics as its main expertise. A number of research projects and prototypes space manipulator were build for space stations. Also it has design a series of light- 
weight UGVs. Machine vision is also among priorities of research in this institute. Lately a new test-field for outdoor UGVs was established within its premises.

Among Russian Universities one should mention following leaders in Robotics.

Bauman Moscow State Technical University is considered to be a leading technical university in Russia. It was one of the very first to teach students robotics. Nowadays Bauman University has several departments in Robotics. In research it has good positions in industrial robotics [10], UGVs, USV, medical robots. As it was mentioned earlier there were quite a few projects made in Bauman which have resulted in real machines being used in Russia and abroad.

MIREA - Russian Technological University is traditionally a center for research in intelligent control algorithms for robots and systems of robots [3]. Lately a brand-new center for industrial robotics "Industry 4.0: Digital Robotic Production" was opened hosting several dozens of various robots and a huge indoor test-field for groups of robots.

Moscow State Technological University "STANKIN" was traditionally specialized in industrial robotics. However the last several years a new turn was done to research and education in medical robotics [8].

Southern Federal University has united several prominent research teams. Speaking about robotics there one should admit that it is a recognized center for multi-agent control of robots due to tradition of a strong research team in Taganrog [6].

Peter the Great St. Petersburg Polytechnic University has joint laboratories together with Russian State Scientific Center for Robotics and Technical Cybernetics (see above). So their research interests mostly coincide.

State University of Aerospace Instrumentation is another University in St.Petersburg who has become very active in robotics. Their specialization is mechatronics and its application in robotics.

Kazan Federal University has introduced robotics recently included robotics into their area of interests. However they have a strong internationally recognized team in intelligent control of robots and another team studying challenging mechanical effects in robotics.

Volgograd State Technical University has prominent positions in walking robots. Actually they have developed several machines which can walk on various types of soil under extreme weather conditions [1].

There are more research teams in universities all over Russia: Skolkovo, Ufa [2], Innopolis, Vladivostok, Kursk, Omsk, Izhevsk and others.

Finalizing this section one should also mention two industrial research centers, which has good reputation in robotics.

Dukhov Automatics Research Institute (VNIIA) is a part of RosAtom Corp. and it is a center for robotics for nuclear industry. It's Robotics Center has produced almost a fifty of various robots mostly for emergencies in nuclear industry.

All-Russian Research Institute for Fire Protection is a part of Russian EMERCOM. It has developed about a dozen of robots for fire fighting and protection.

\section{View for a future}

Speaking of the future trends Russian roboticists foresee following two trends: on one hand there will be a growing demand to increase a number of robots to be usable and affordable for common people. And on the other hand upcoming global financial transformation may cause serious shortcuts of funding for design and production of effective robots. However we consider that we should keep our efforts to produce new effective machines.

As for general directions for research in robotics we see the following:

New scenarios and applications should be searched and developed for further robot dissemination. We consider it as one of typical mistakes when people consider robot as a 
machine which just replaces human at fulfilling some job. In fact quite often robot can not just replace human but moreover it may perform some operation completely new way or even perform an operation impenetrable for a human.

Increase of robots' effectiveness. Frankly speaking we must admit that still a lot of robots available for end-users do not make their end-users completely happy. We must search for new ways to decrease their life-cycle costs and increase quality and effectiveness of operation. As a fact we may admit that modern robotics gives a huge field for further optimization.

Increase of robots' autonomy is another challenge. We understand that in fact a lot of robots' usages still imply teleoperation with an active participation of human as an operator. In some applications this is done concisely (medicine, military) as an ethical (or even juridical) response, in other applications this is caused by a shortage of robots' "intelligence".

Multi-robot application is a new challenge. In fact a lot has been done in this area lately. However most of the research was concentrated at control aspects of this problem. We consider that we should have a more general overview on $\mathrm{HOW}$ robots will be used in groups. But of course this has a strong restriction by low autonomy level of modern robots.

Improvement of human - robot interaction. Actually this topic has a double meaning. On one side as soon as operator is still an important player of robotic system we must improve information presentation to operator, improve mechanical contact of operator with robot (where applicable). Another meaning is in psychological acceptance of robots by humans (here we mean not only operator but all humans present in robots' environment).

Speaking of international cooperation we must admit that there is a series of sources available for funding. E.g. Ministry of Science and Higher Education, Russian Fund of Basic Research, Russian Science Foundation and some others. There is a number of bilateral agreements between Russian Federation and other countries which may support joint research in various areas including robotics.

\section{Conclusion}

As a conclusion we must admit that Russian robotics has a long-term history, impressive present and challenging future. Because of this we believe that our scientists are a good part of band of world roboticists.

\section{Acknowledgments}

This work was partially supported by the Ministry of Science and Higher Education within the framework of the Russian State Assignment under contract No. AAAA-A20120011690138-6.

\section{References}

1. Briskin, E.S., Kalinin, Y.V., Miroshkina, M.V., Energy Efficient Modes of the Motion of Mobile Robots with Orthogonal Stepping Motors when Overcoming Obstacles // Journal of Computer and Systems Sciences International 59(2), (2020).

2. Darintsev O.V., Judintsev B.S., Alekseev A.Ju. Methods of a Heterogeneous Multiagent Robotic System Group Control // Procedia Computer Science. - Vol. 150. (2019).

3. Diane, S.A.K., Manko, S.V., Margolin, I.D., Novoselskiy, A.K. Hierarchical scenarios for behavior planning in autonomous robots, Proceedings of the 2019 IEEE Conference of Russian Young Researchers in Electrical and Electronic Engineering, ElConRus (2019).

4. Ermolov I. Hierarchical Data Fusion Architecture for Autonomous Systems// ACTA IMEKO. - 2019. - Vol. 8, no. 4.

5. Ermolov I. Industrial robotics review // Robotics: Industry 4.0 Issues \& New Intelligent Control Paradigms. - Vol. 272 of Studies in Systems, Decision and Control. — Springer, Cham, (2020).

6. Kalyaev, I., Kapustyan, S., Ivanov, D. et al, A novel method for distribution of goals among UAVs for oil field monitoring 2017 Proceedings of the 6th International Conference on 
Informatics, Electronics and Vision and 2017 7th International Symposium in Computational Medical and Health Technology, ICIEV-ISCMHT (2017).

7. Laryushkin Pavel, Glazunov Victor and Demidov Sergey, Singularity Analysis of 3-DOF Translational Parallel Manipulator, in Advances on Theory and Practice of Robots and Manipulators, Proceedings of ROMANSY 2014 XX CISM-IFToMM Symposium on Theory and Practice of Robots and Manipulators, (2014).

8. Levin A.A, Klimov D.D., Nechunaev A.A., Vorotnikov A.A., Prokhorenko L.S., Grigorieva E.V., Astakhov D.A., Poduraev Y.V., Panchenkov D.N. The comparison of the process of manual and robotic positioning of the electrode performing radiofrequency ablation under the control of a surgical navigation system. Scientific Reports 10, 8612 (2020).

9. Pavliuk N., Smirnov P., Kondratkov A., Ronzhin A. Connecting Gripping Mechanism Based on Iris Diaphragm for Modular Autonomous Robots // International Conference on Interactive Collaborative Robotics. (2019).

10. Shereuzhev M., Serebrenny V. Industrial Collaborative Multi-agent Systems: Main Challenges. In: Ronzhin A., Shishlakov V. (eds) Proceedings of 14th International Conference on Electromechanics and Robotics "Zavalishin's Readings". Smart Innovation, Systems and Technologies, vol 154. Springer, Singapore (2020).

11. VaulinYu.V., Dubrovin F.S., Scherbatyuk, A.F., Some Algorithms for Determining an Unknown Initial Position of AUV Using Information from a Single Beacon Navigation System, Gyroscopy and Navigation, 8(3), (2017).

12. V. G. Gradetsky, M. M. Knyazkov, E. A. Semenov, A. N. Sukhanov//Structure of wall climbing robot control system / 2019 IEEE International Symposium on Measurement and Control in Robotics (ISMCR). — 8955718. — IEEE Houston, TX, USA, (2019). 